\title{
As adaptações na capacidade aeróbia não são transferidas para a capacidade de sprints repetitivos
}

http://dx.doi.org/10.11606/1807-5509202000030365
Carlos Augusto Kalva-Filho* João Paulo Loures* Paulo Eduardo Redkva** Alessandro Moura Zagatto*** Marcelo Papoti****

\section{Resumo}

No presente estudo, objetivou-se testar se a melhora da capacidade aeróbia, observada após oito semanas de treinamento, é transferida para a capacidade de sprints repetitivos (CSR). Dez corredores (18 \pm 1 ano; estatura de 170,1 $\pm 8,0 \mathrm{~cm}$; massa corporal de 66,4 $\pm 6,6 \mathrm{~kg}$ ) participaram do estudo. A capacidade aeróbia foi considerada como a intensidade correspondente à concentração de $4 \mathrm{mM}$ de lactato (OBLA), determinada durante teste incremental, realizado em pista de atletismo. A capacidade de sprints repetitivos foi avaliada por meio de seis sprints de $35 \mathrm{~m}$, separados por dez segundos de intervalo passivo. Todos os testes foram aplicados antes e após oito semanas de treinamento polarizado. Os efeitos do treinamento foram evidenciados por meio do teste $t$ de Student para amostras dependentes, e as possiveis relações entre as alterações percentuais do OBLA e a CSR foram verificadas pelo teste de correlação de Pearson. Para todas as análises o nível de significância foi de $p<0,05$. Após o treinamento, todos os participantes apresentaram aumento significativo da intensidade de OBLA (antes 15,4 $\pm 0,9$ $\mathrm{km} \cdot \mathrm{h}^{-1}$; após $\left.17,2 \pm 1,6 \mathrm{~km} \cdot \mathrm{h}^{-1} ; p=0,01\right)$. Na avaliação da CSR, constatou-se que a velocidade máxima, a velocidade média e 0 índice de fadiga não foram modificados com o treinamento $(p>0,61)$. Nenhuma correlação foi observada entre as alterações percentuais da intensidade de OBLA e as alterações das variáveis relacionadas à capacidade de sprints repetitivos $(r<-0,26 ; p>0,47)$. Assim, esses resultados demonstram que a melhora da capacidade aeróbia não é transferida para a CSR, avaliada por meio de seis esforços, separados por dez segundos de intervalo passivo.

Palavras-chave: Treinamento; OBLA; Corredores; Velocidade Média.

\section{Introdução}

A habilidade de realizar esforços de alta intensidade e curta duração $(<10 \mathrm{~s})$, separados por curtos períodos de recuperação, é denominada Capacidade de Sprints Repetitivos (CSR) ${ }^{1}$. Durante os períodos de esforço máximo, a demanda energética é suprida, principalmente, pelos metabolismos anaeróbios (alático e lático), com menor participação do metabolismo aeróbio ${ }^{1}$.

Entretanto, durante os períodos de recuperação, o metabolismo aeróbio é predominante, sobretudo para que ocorra a remoção de fosfatos inorgânicos intracelulares $(\mathrm{Pi})$ e a recuperação dos estoques de Fosfocreatina (PCr), fatores essenciais para a CSR ${ }^{1,2}$.
Por meio desse panorama fisiológico, é possível explicar as significativas correlaçôes entre a CSR e parâmetros relacionados ao metabolismo aeróbio (por exemplo, consumo máximo de oxigênio; $\left.\mathrm{VO}_{2 \mathrm{MAX}}\right)^{3-6}$, sugerindo-se que o treinamento aeróbio pode potencializar a recuperação do metabolismo anaeróbio alático entre os esforços, melhorando, consequentemente, a CSR ${ }^{7}$.

De fato, diferentes estudos têm investigado as transferências da aptidão aeróbia, induzidas pelo treinamento, para a $\mathrm{CSR}^{7-10}$. Entretanto, a melhora do metabolismo aeróbio, nesses estudos, tem sido atribuída ao aumento dos valores de
*Faculdade de Medicina de Ribeirão Preto, Universidade de São

Paulo, Ribeirão Preto, SP, Brasil.

${ }^{* *}$ Instituto de Biociências, Universidade Estadual Paulista, Rio Claro, SP, Brasil.

***Universidade Estadual Paulista, Bauru, SP, Brasil.

${ }^{* * * *}$ Escola de Educação Física e Esportes de Ribeirão Preto, Universidade de São Paulo, Ribeirão Preto, SP, Brasil. 
$\mathrm{VO}_{2 \mathrm{MAX}}$, condição limitada para atletas de alto nível, e fortemente dependente de fatores centrais (por exemplo, transporte sanguíneo de $\left.\mathrm{O}_{2}\right)^{11}$. Considerando que a manutenção do desempenho, em esforços consecutivos, é dependente de fatores periféricos ${ }^{12}$, a utilização do $\mathrm{VO}_{2 \mathrm{MAX}}$ pode limitar o entendimento sobre as reais transferências do treinamento aeróbio para a CSR. Diferentemente, pela capacidade aeróbia, isto é, limiar anaeróbio, é possível explicar, de maneira mais satisfatória, como as adaptaçōes aeróbias são transferidas para a CSR, principalmente por também estar relacionada a fatores periféricos ${ }^{13}$. Entretanto, em poucos estudos testou-se se as adaptações observadas na capacidade aeróbia são, de fato, transferidas para a CSR.

Além disso, na maioria dos estudos em que se investigou a transferência das adaptaçōes aeróbias para a CSR, foram utilizadas sessões de treinamento aplicadas com intensidades exclusivamente abaixo do limiar anaeróbio ${ }^{7,10}$ ou próximas ao $\mathrm{VO}_{2 \mathrm{MAX}}{ }^{8}$. Nesse contexto, o treinamento polarizado é considerado importante estratégia para a distribuição das intensidades, ao longo da temporada ${ }^{14,15}$. Com base em tal estratégia de distribuição, o treinamento é realizado com diferentes intensidades, as quais são relativas tanto ao limiar anaeróbio como àquelas próximas à intensidade correspondente ao $\mathrm{VO}_{2 \mathrm{MAX}}{ }^{14,15}$. Considerando-se que o treinamento polarizado tem apresentado eficácia para o aumento da capacidade aeróbia ${ }^{15}$, é possível que esse modelo também aumente a CSR, perspectiva ainda pouco explorada na literatura'.

Outro aspecto, que igualmente necessita mais investigações, está relacionado aos protocolos utilizados para avaliação da CSR e suas possíveis influências sobre as transferências aeróbias. De fato, nos estudos anteriores em que se avaliou a CSR, após períodos de treinamento aeróbio, foram utilizados protocolos com muitos esforços e/ou longos períodos de recuperação ${ }^{7-10}$, o que, sabidamente, aumenta as contribuiçôes do metabolismo aeróbio ${ }^{12}$. Nesse contexto, as possíveis transferências da capacidade aeróbia para a CSR, avaliada por meio de poucos esforços, separados por curtos períodos de recuperação, ainda não foram demonstradas na literatura.

Assim, no presente estudo, objetivou-se testar se a melhora da capacidade aeróbia, observada após oito semanas de treinamento polarizado, é transferida para a CSR, avaliada por meio de seis esforços máximos, separados por dez segundos de intervalo passivo.

\section{Método}

\section{Participantes}

Participaram deste estudo dez corredores do sexo masculino, especialistas na prova do facho (11,6 km; $970 \mathrm{~m}$ por atleta), com idade média de $18 \pm 1$ ano, estatura de $170,1 \pm 8,0 \mathrm{~cm}$ e massa corporal de $66,4 \pm 6,6 \mathrm{~kg}$. Todos os atletas foram informados a respeito dos riscos e benefícios do presente estudo; foram incluídos nas análises somente aqueles que concordaram e assinaram o Termo de Consentimento Livre e Esclarecido. Os procedimentos deste estudo foram aprovados pelo Comitê de Ética em Pesquisa da Universidade Federal do Mato Grosso do Sul (no 146/06) e conduzidos conforme os princípios da Declaração de Helsinki.

\section{Delineamento experimental}

Os participantes foram submetidos a período de treinamento de oito semanas, com o objetivo

de aumentar sua capacidade aeróbia. Antes e após o período de treinamento, as avaliações foram realizadas em duas visitas, com intervalo mínimo de 24 horas. Na primeira visita, os atletas foram submetidos a teste incremental em pista oficial de atletismo, para determinação da intensidade correspondente à concentração de $4 \mathrm{mM}$ (Onset of Blood Lactate Accumulation - OBLA), a qual, posteriormente, foi utilizada para a prescrição das sessôes de treinamento e assumida como índice de capacidade aeróbia. Na segunda visita, a CSR foi avaliada também em pista de atletismo.

\section{Determinação da capacidade aeróbia}

Para determinação do OBLA, os participantes realizaram protocolo incremental, em pista oficial de atletismo, que consistiu em esforços de $800 \mathrm{~m}$, com velocidade inicial de $11 \mathrm{~km} \cdot \mathrm{h}^{-1}$ e incrementos de 1 $\mathrm{km} \cdot \mathrm{h}^{-1}$ até o quinto estágio $\left(16 \mathrm{~km} \cdot \mathrm{h}^{-1}\right)$, a partir do qual os avaliados foram instruídos a realizar esforço 
máximo de $800 \mathrm{~m}$. A velocidade foi controlada por estímulos sonoros a cada $100 \mathrm{~m}$ e, em todos os estágios, o tempo real de esforço foi mensurado, para posterior correção da intensidade, se necessário.

Imediatamente ao final de cada estágio, foram coletados $25 \mu \mathrm{l}$ de sangue do lóbulo da orelha, para determinação das concentrações sanguíneas de lactato ([La-]), utilizando-se lactímetro eletroquímico (Yellow Springs Instruments, modelo 1500 Sport, Ohio, USA). O OBLA foi considerado como a intensidade correspondente a $4 \mathrm{mM}$, obtida por meio da relação exponencial entre [ $\left.\mathrm{La}^{-}\right] \mathrm{e}$ intensidade de exercício ${ }^{16}$. Tais ajustes matemáticos foram realizados por meio de software específico (OriginPro 7.0, Microcal, MA, USA).

\section{Avaliação da CSR}

A CSR foi avaliada por meio de seis esforços máximos de $35 \mathrm{~m}$, intercalados por período de recuperação passiva de dez segundos. $\mathrm{O}$ registro de tempo foi realizado a cada esforço, pelo sistema de fotocélula CEFISE, localizado no início e final do percurso. Como variáveis relacionadas à CSR, foram determinadas a velocidade máxima $\left(\mathrm{V}_{\mathrm{MAX}}\right)$, a velocidade média $\left(\mathrm{V}_{\mathrm{MED}}\right)$ e o índice de fadiga (IF) durante os seis esforços $(\mathrm{IF}(\%)=((\mathrm{PP}-$ Potência Mínima)x100)/PP)). Esse modelo de avaliação da CSR foi escolhido por apresentar elevados índices de reprodutibilidade ${ }^{17,18}$.

\section{Treinamento polarizado}

O treinamento teve duração de oito semanas, com frequência semanal de cinco sessões, totalizando 40 sessões, com volume médio de $8,0 \pm 3,3 \mathrm{~km} \cdot \mathrm{dia}^{-1}$. A intensidade, em todas as sessões, foi controlada por meio do tempo necessário para distância da série.

Três zonas de treinamento, relativas à intensidade de OBLA, foram aplicadas. As sessóes realizadas abaixo do OBLA (Z1) tiveram volume médio de $29,7 \pm 6,5 \mathrm{~km} \cdot \mathrm{semana}^{-1}$, correspondendo a $68 \%$ do volume total. Os atletas realizaram $12 \%$ das sessôes de treinamento (volume médio de 8,4 \pm $3,1 \mathrm{~km} \cdot \mathrm{semana}^{-1}$ ) com intensidade correspondente ao OBLA (Z2). As sessões realizadas acima do OBLA (Z3) tiveram volume médio de 5,6 $\pm 2,1$ $\mathrm{km} \cdot \mathrm{semana}^{-1}$, correspondendo a $20 \%$ do volume total (FIGURA 1).

No volume realizado em Z3, compreenderamse estímulos aeróbios de alta intensidade (13\% do volume total), com intensidade correspondente a $110 \%$ do OBLA, e estímulos anaeróbios $(7 \%$ do volume total), nos quais houve realização de esforço máximo. As sessões de treinamento aeróbio de alta intensidade foram aplicadas utilizando-se distâncias entre 400 e 1200 m, bem como intervalos entre as repetições de 60 a 180 segundos, caracterizando razões esforço-pausa de $\approx 1: 1$. Os atletas realizaram de duas a três sessões de treinamento em $\mathrm{Z} 3$ por semana.

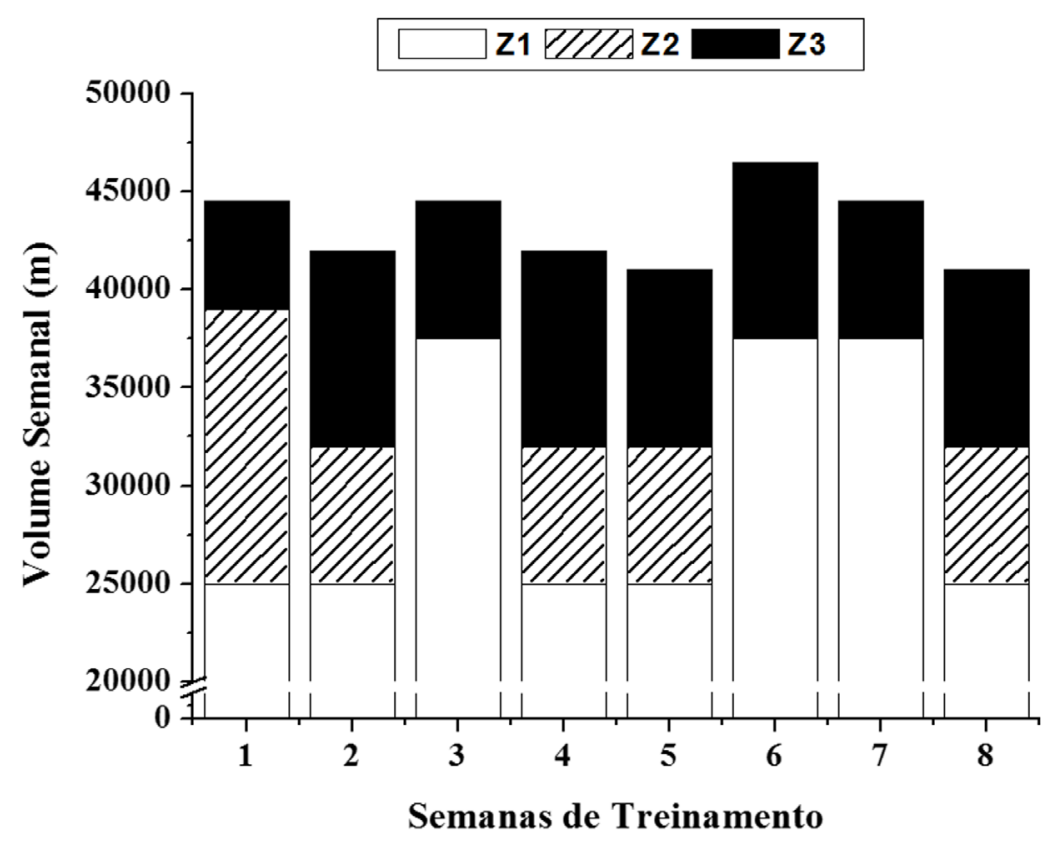

FIGURA 1 -Distribuição das zonas de treinamento abaixo $(\mathrm{Z} 1)$ da intensidade de limiar anaeróbio (OBLA), no OBLA (Z2) e acima do OBLA (Z3) durante as oito semanas de treinamento 


\section{Tratamento estatístico}

A normalidade dos dados foi testada e confirmada pelo teste de Shapiro-Wilk, o qual possibilitou análise estatística paramétrica e apresentação dos resultados em média \pm desviopadrão. Para comparação entre as variáveis obtidas antes e após o período de treinamento, foi utilizado o teste $t$ de Student para amostras dependentes, acompanhado pelos valores de effect size (ES), os quais foram classificados como muito pequeno $(<0,2)$, pequeno $(0,21$
$-0,5)$, médio $(0,51-0,8)$ e grande $(>0,81)^{19}$. Para determinação das possíveis transferências da capacidade aeróbia para a CSR, o teste de correlação de Pearson foi aplicado sobre as diferenças percentuais observadas após o período de treinamento tanto para o OBLA quanto para os parâmetros obtidos com os esforços repetitivos (e.g., $\Delta \%=(($ Valor pósx 100$) /$ Valor pré)-100). Todas as análises foram realizadas com auxílio do pacote estatístico STATISTICA 7 (Statsoft, EUA) e, em todos os casos, o nível de significância foi fixado em $p<0,05$.

\section{Resultados}

Na TABELA 1 mostram-se os valores obtidos antes e após o treinamento. Todos os corredores apresentaram aumento da intensidade relativa ao OBLA, após o treinamento polarizado (ES grande). Entretanto, as variáveis relacionadas à CSR não foram alteradas após o treinamento (ES muito pequeno ou pequeno). A FIGURA 2 demonstra, em valores individuais, a diferença percentual da capacidade aeróbia e das variáveis provenientes relacionadas à CSR. Nenhuma correlação foi constatada entre a melhora percentual do OBLA e as diferenças percentuais observadas para a CSR após o período de treinamento (TABELA 2).
ES: effect size; OBLA: Onset of Blood Lactate Accumulation (capacidade aeróbia correspondente à concentração sanguínea de lactato de $4 \mathrm{mM}$ ); $\mathrm{V}_{\text {MAX }}$ : velocidade máxima; $\mathrm{V}_{\text {MED }}$ : velocidade média; IF: índice de fadiga.
TABELA 1 -Valores médios \pm desvio-padrão e comparações entre os parâmetros, avaliados antes e após oito semanas de treinamento

\begin{tabular}{ccccc}
\hline & Antes & Após & $p$ & ES \\
\hline OBLA $\left(\mathrm{km} \cdot \mathrm{h}^{-1}\right)$ & $15,4 \pm 0,9$ & $17,2 \pm 1,6$ & 0,01 & 1,45 \\
$\mathrm{~V}_{\text {MAX }}(\mathrm{m} / \mathrm{s})$ & $7,2 \pm 0,5$ & $7,2 \pm 0,3$ & 0,89 & 0,07 \\
$\mathrm{~V}_{\text {MED }}(\mathrm{m} / \mathrm{s})$ & $6,7 \pm 0,4$ & $6,8 \pm 0,2$ & 0,63 & 0,21 \\
$\mathrm{IF}(\%)$ & $30,2 \pm 13,0$ & $27,5 \pm 11,7$ & 0,62 & 0,22 \\
\hline
\end{tabular}
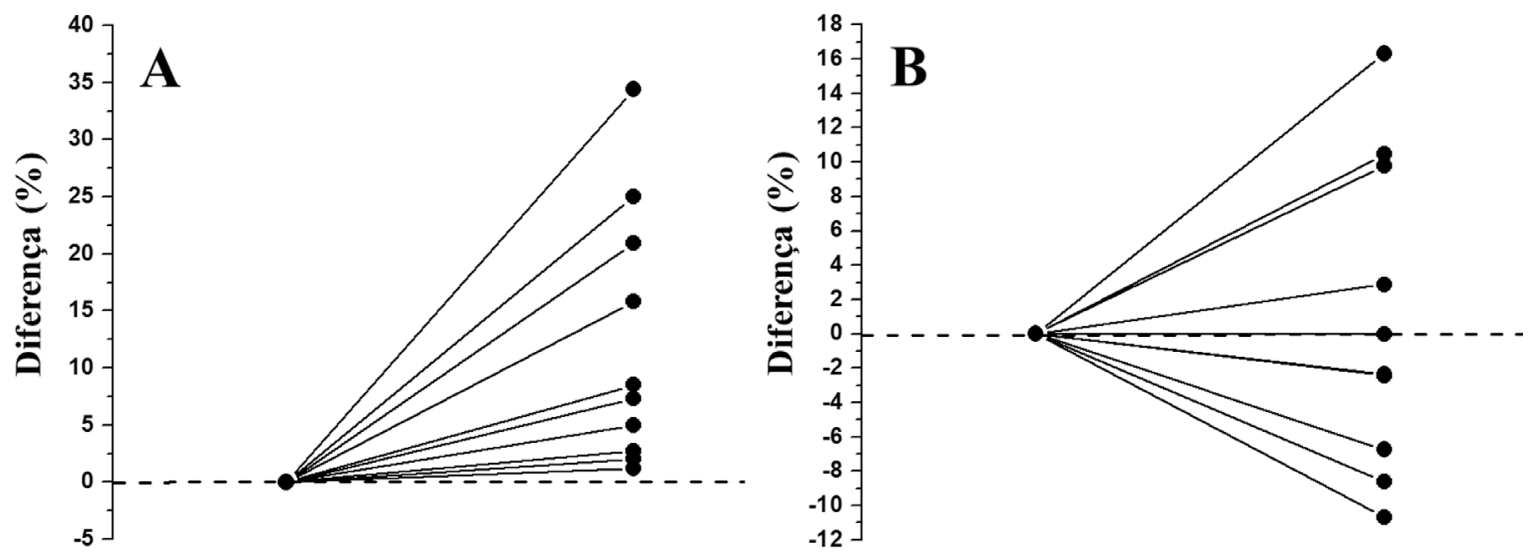

FIGURA 2 - Diferença percentual individual observada com o treinamento para a capacidade aeróbia (A), velocidade máxima (B), velocidade média (C) e o índice de fadiga (D).

continua 

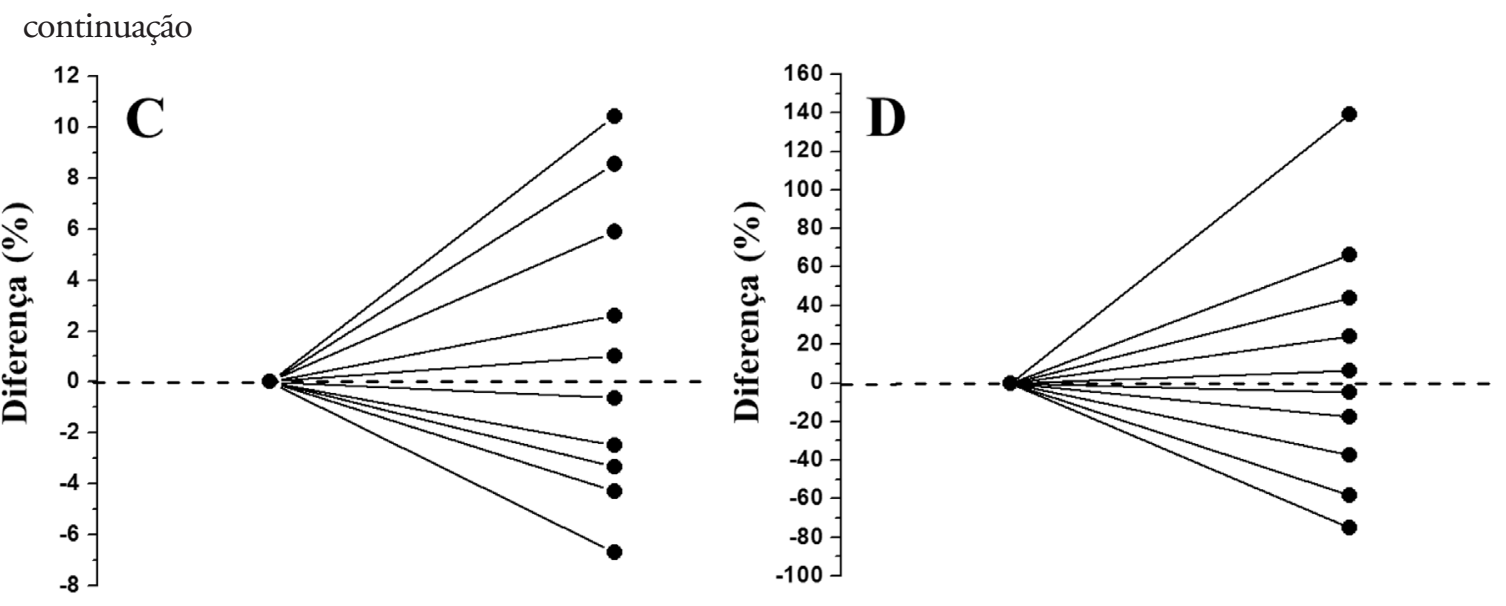

TABELA 2 -Correlações entre as alterações percentuais $(\Delta \%)$ da capacidade aeróbia e as variáveis relacionadas à capacidade de sprints repetitivos

\begin{tabular}{ccc}
\hline & $\mathrm{r}$ & $\mathrm{p}$ \\
\hline VMAX & 0,03 & 0,93 \\
VMED & 0,05 & 0,90 \\
IF & 0,08 & 0,83 \\
\hline
\end{tabular}

$\mathrm{V}_{\text {MAX }}:$ velocidade máxima; $\mathrm{V}_{\mathrm{MED}}$ : velocidade média; IF: índice de fadiga.

\section{Discussão}

Os principais achados presentes neste estudo demonstram que a melhora na capacidade aeróbia não é transferida para a CSR, avaliada por meio de seis esforços máximos de $35 \mathrm{~m}$, separados por dez segundos de intervalo passivo.

EDGE et al. ${ }^{7}$ compararam os efeitos de dois modelos de treinamento sobre as adaptaçóes da capacidade aeróbia e CSR. Os treinamentos consistiram em estímulos de alta intensidade (6-10 x $2 \mathrm{~min}$, com intensidades entre $120 \mathrm{e}$ $140 \%$ do limiar anaeróbio) e de baixa intensidade (20 - 30 min contínuos, com intensidades entre 80 e $95 \%$ do limiar anaeróbio), ambos efetivos para o aumento da capacidade aeróbia ${ }^{7}$. Embora a CSR também tenha sido potencializada por ambos os treinamentos, as respostas observadas no grupo que treinou em alta intensidade foram maiores do que as observadas no grupo de baixa intensidade. Similarmente, McGinley e Bishop $^{8}$ observaram que o treinamento, quando realizado em intensidades próximas do $\mathrm{VO}_{2 \mathrm{MAX}}$, é mais efetivo para o aumento da CSR e induz alteraçôes significativas na capacidade aeróbia. Em conjunto, nos estudos supracitados ${ }^{7,8}$, demonstra-se que as adaptaçóes na CSR não ocorrem concomitantemente ao aumento da capacidade aeróbia, concordando com os achados da presente investigação.

Nesse contexto, além da capacidade aeróbia, as capacidades de tamponamento e de reoxigenação muscular podem ser igualmente determinantes para a $\operatorname{CSR}^{8,9}$. Em relação à capacidade de tamponamento muscular, tem-se demonstrado que estímulos acima do limiar anaeróbio são mais efetivos para induzir adaptaçóes sobre essa variável, em relação ao treinamento em baixa intensidade ${ }^{20}$. Além disso, BuchHeit e Ufland ${ }^{9}$ observaram correlaçôes significativas entre as alterações da capacidade de reoxigenação muscular e a CSR, observadas após oito semanas de treinamento polarizado (83\% em Z1 ou Z3 e $17 \%$ em Z3). Assim, recomenda-se que sessões de alta intensidade sejam implementadas durante o treinamento, a fim de induzir adaptações positivas sobre a capacidade de tamponamento e de reoxigenação muscular, ambas relacionadas à CSR.

A distribuição de intensidades proposta neste estudo (68\% em Z1, 12\% em Z2 e 20\% em Z3) é similar à realizada em estudos prévios ${ }^{15,21}$, sendo 
eficaz para a melhora da capacidade aeróbia dos participantes em $\approx 12 \pm 11 \%(1,2-34,4 \%)$. Tais resultados estão de acordo com as constatações de STÖggl e Sperlich ${ }^{15}$, nas quais observaramse aumentos no OBLA, após nove semanas de treinamento polarizado $(68 \% \mathrm{em} \mathrm{Z1,6 \%} \mathrm{em}$ Z2 e $26 \%$ em Z3). Além disso, considerando o fato de a capacidade de tamponamento muscular apresentar adaptações, após pequenos períodos de treinamento em alta intensidade (6 sessões em $Z 3 ;^{22}$ ), e a capacidade de reoxigenação ser potencializada pelo treinamento polarizado $\left(\approx 20 \%\right.$ em $\left.\mathrm{Z3} ;{ }^{\circ}\right)$, é plausível que essas variáveis tenham melhorado após a intervenção aplicada neste estudo. Entretanto, nos resultados, aqui, demonstra-se que essas possíveis adaptações não foram transferidas para a CSR.

Nesse sentido, embora o modelo de esforço utilizado para avaliação da CSR apresente reprodutibilidade e validade satisfatórias ${ }^{17,18}$, o número de esforços e o intervalo aplicado entre cada exercício (6x35 m separados por dez segundos) foram significativamente diferentes de estudos anteriores. De fato, neste estudo, testou-se pela primeira vez as transferências da capacidade aeróbia para a CSR, por meio de poucos esforços, separados por curtos intervalos de recuperação. Considerando o fato de a participação do metabolismo aeróbio, durante esforços repetitivos, ser extremamente influenciada pelo intervalo e número de esforços realizados ${ }^{12}$, as diferenças entre os protocolos utilizados corroboram resultados observados no presente estudo.

No contexto em questão, nos estudos anteriores, em que foram observadas melhoras após o treinamento predominantemente aeróbio na CSR, avaliou-se tal habilidade por meio da aplicação de intervalos maiores que 24 segundos entre os esforços ${ }^{7,8,10,20}$. Com esses intervalos, a recuperação dos estoques de $\mathrm{PCr}$, embora não tenha sido completa, foi maior do que durante os dez segundos de recuperação passiva aplicados na presente investigação. De fato, em apenas um estudo demonstrou-se a sensibilidade da CSR, avaliada com intervalos de dez segundos entre os esforços. Para tanto, GlaIsTER et al. ${ }^{10}$ utilizam 20 esforços de cinco segundos, o que, sabidamente, aumenta a contribuição do metabolismo aeróbio, especialmente durante os últimos esforços ${ }^{12}$. Desse modo, embora seja protocolo reprodutível e válido para seu propósito, na avaliação da CSR, por meio de apenas seis esforços, separados por dez segundos, as transferências do metabolismo aeróbio podem ser comprometidas, informação essa apresentada pela primeira vez no presente estudo.

Essa afirmação é corroborada pelos achados de Milioni et al. ${ }^{23}$, nos quais foi utilizado protocolo idêntico ao aplicado no presente estudo, para avaliação da CSR. Esses autores observaram que a contribuição do metabolismo aeróbio foi de $40 \%$ durante todo o protocolo (isto é, esforços e períodos de recuperação) e de apenas $20 \%$ durante os esforços. Assim, considerando as pequenas contribuições aeróbias para que a CSR, avaliada por seis esforços, separados por dez segundos, apresente sensibilidade ao treinamento, é possível que sejam necessários maiores volumes de estímulos anaeróbios, os quais representam apenas $7 \%$ das sessões aplicadas, no presente estudo. De fato, alguns participantes apresentaram aumento dos valores de $V_{\text {MAX }}, V_{\text {MED }}$ e IF, o que pode ser relacionado ao pequeno volume de treinamento anaeróbio, principalmente pelas correlaçôes insignificantes entre essas alterações e aquelas observadas para a capacidade aeróbia. Assim, o treinamento proposto proporcionou aumento da capacidade aeróbia, cumprindo o principal objetivo no presente estudo, mas não foi eficaz para a melhora significativa da CSR, avaliada pelo protocolo utilizado.

Embora tenha-se respeitado a especificidade e o ambiente de treinamento/competição dos corredores, a principal limitação, neste estudo, foi a utilização de protocolo ainda não validado para determinação do OBLA. Desse modo, em estudos futuros, é necessário que os presentes resultados sejam confirmados, utilizando-se métodos mais robustos para a determinação da capacidade aeróbia. Além disso, a fim de confirmar a hipótese de que o intervalo entre os esforços possui papel determinante para as transferências da capacidade aeróbia, outros modelos de esforço repetitivo devem ser comparados ao utilizado neste estudo.

Desse modo, no presente estudo, demonstrouse que o treinamento polarizado é efetivo para aumentar a capacidade aeróbia de corredores. Entretanto, essas adaptaçôes não foram transferidas para a CSR, avaliada por meio de seis esforços, separados por dez segundos de intervalo passivo. 


\title{
Agradecimentos
}

Aos Técnicos e atletas, que permitiram a realização deste estudo e foram voluntários. Ao professor Edson Itaru Kaminagakura (in memoriam), pela paciência e Cooperação.

\section{Conflito de interesse}

Declara-se não haver conflitos de interesse entre os autores.

\begin{abstract}
Adaptations in aerobic capacity are not transferred to the repeated sprint ability

The aim of the present study was to determine if the improvement in aerobic capacity observed after eight weeks of training is transferred to the repeated sprint ability. Ten runners ( $18 \pm 1$ years, height of 170.1 $\pm 8.0 \mathrm{~cm}$ and body mass of $66.4 \pm 6.6 \mathrm{~kg}$ ) participated in this study. The aerobic capacity was considered as the intensity corresponding to blood lactate concentrations of $4 \mathrm{mM}(\mathrm{OBLA})$, determined during an incremental test performed in an athletics track. RSA was evaluated using six all-out sprints of $35 \mathrm{~m}$, separated by $10 \mathrm{~s}$ of passive recovery. All tests were applied before and after eight weeks of polarized training. Training effects were evidenced using student's t-test for repeated measures and the possible correlations were tested using Pearson's test. For all analysis, the significance level was set at $p<0.05$. All participants presented significant improvements for OBLA intensity (before $15,4 \pm 0,9 \mathrm{~km} \cdot \mathrm{h}^{-1}$; after 17,2 $\left.\pm 1,6 \mathrm{~km} \cdot \mathrm{h}^{-1} ; p=0,01\right)$. The RSA assessments demonstrated no effects for maximal speed, mean speed and fatigue index obtained during RSA test $(p>0,61)$. No correlations were observed between percentage changes in the intensity of OBLA and changes in the variables related to RSA $(r<-0,26 ; p>0,47)$. Thus, these results demonstrate that the improvement in aerobic capacity is not transferred for RSA measured throw six sprints separated by ten seconds of passive recovery.
\end{abstract}

KEYwords: Aerobic Training; OBLA; Runners; Mean Velocity.

\section{Referências}

1. Girard O, Mendez-Villanueva A, Bishop D. Repeated-sprint ability - Part I. Sports Med. 2011;41(8):673-694.

2. Glaister M. Multiple sprint work - physiological responses, mechanisms of fatigue and the influence of aerobic fitness. Sports Med. 2005;35(9):757-777.

3. Andrade VLd, Kalva-Filho CA, Zagatto AM, Kaminagakura EI, Papoti M, Santiago PRP. Influence of aerobic fitness in running anaerobic sprint test (RAST). Motriz. 2013;19(3):1-7.

4. Gharbi Z, Dardouri W, Haj-Sassi R, Chamari K, Souissi N. Aerobic and anaerobic determinants of repeated sprint ability in team sports athletes. Biol Sport. 2015;32(3):207-212.

5. Kalva-Filho CA, Loures JP, Franco VH, Kaminagakura EI, Zagatto AM, Papoti M. Correlaçóes entre parâmetros aeróbios e desempenho em esforços intermitentes de alta intensidade. Motriz. 2013;19(2):306-312.

6. Baldi M, Da Silva J, Buzachera C, Castagna C, Guglielmo L. Repeated sprint ability in soccer players: Associations with physiological and neuromuscular factors. J Phys Fit Sports Med. 2016;57(2):26-32.

7. Edge J, Bishop D, Goodman C, Dawson B. Effects of high-and moderate-intensity training on metabolism and repeated sprints. Med Sci Sports Exerc. 2005;37(11):1975-1982.

8. McGinley C, Bishop DJ. Influence of training intensity on adaptations in acid/base transport proteins, muscle buffer 
capacity, and repeated-sprint ability in active men. J Appl Physiol. 2016;121(6):1290-1305.

9. Buchheit M, Ufland P. Effect of endurance training on performance and muscle reoxygenation rate during repeated-sprint running. Eur J Appl Physiol. 2011;111(2):293-301.

10. Glaister M, Stone MH, Stewart AM, Hughes MG, Moir GL. The influence of endurance training on multiple sprint cycling performance. J Strength Cond Res. 2007;21(2):606-612.

11. Bassett DR, Howley ET. Limiting factors for maximum oxygen uptake and determinants of endurance performance. Med Sci Sports Exerc. 2000;32(1):70-84

12. Spencer M, Bishop D, Dawson B, Goodman C. Physiological and metabolic responses of repeated-sprint activities. Sports Med. 2005;35(12):1025-2044.

13. Billat V, Sirvent P, Koralsztein J, Mercier J. The concept of maximal lactate steady state: A bridge between biochemistry, physiology and sports science. Sport Med. 2003;33(6):407-426.

14. Hydren JR, Cohen BS. Current scientific evidence for a polarized cardiovascular endurance training model. J Strength Cond Res. 2015;29(12):3523-3530.

15. Stöggl T, Sperlich B. Polarized training has greater impact on key endurance variables than threshold, high intensity, or high volume training. Front Physiol. 2014;5:33.

16. Heck H, Mader A, Hess G, Mücke S, Müller R, Hollmann W. Justification of the 4-mmol/l lactate threshold. Int J Sports Med. 1985;6(3):117-130.

17. De Andrade VL, Pereira Santiago PR, Kalva Filho CA, Zapaterra Campos E, Papoti M. Reproducibility of Running Anaerobic Sprint Test for soccer players. J Phys Fit Sports Med. 2016;56(1-2):34-38.

18. Zagatto AM, Beck WR, Gobatto CA. Validity of the running anaerobic sprint test for assessing anaerobic power and predicting short-distance performances. J Strength Cond Res. 2009;23(6):1820-1827.

19. Cohen J. Statistical power analysis for the behavioral sciences. Hillsdale: Lawrence Earlbaum Associates; 1988. p. 2026.

20. Edge J, Bishop D, Goodman C. The effects of training intensity on muscle buffer capacity in females. Eur J Appl Physiol. 2006;96(1):97-105.

21. Muñoz I, Seiler S, Bautista J, España J, Larumbe E, Esteve-Lanao J. Does polarized training improve performance in recreational runners? Int J Sports Physiol Perform. 2014;9(2):265-272.

22. Weston AR, Myburgh KH, Lindsay FH, Dennis SC, Noakes TD, Hawley JA. Skeletal muscle buffering capacity and endurance performance after high-intensity interval training by well-trained cyclists. Eur J Appl Physiol Occup Physiol. 1996;75(1):7-13.

23. Milioni F, Zagatto AM, Barbieri R, Andrade V, dos Santos JW, Gobatto CA, et al. Energy systems contribution in the running-based anaerobic sprint test. Int J Sports Med. 2017;38(3):226-232. 